\title{
NONHOLONOMIC MOBILE ROBOT WITH DIFFERENTIAL CHASSIS MATHEMATICAL MODELLING AND IMPLEMENTATION IN SIMULINK WITH FRICTION IN DYNAMICS
}

\author{
Jakub ČERKALA, Anna JADLOVSKÁ \\ Department of Cybernetics and Artificial Intelligence, Faculty of Electrical Engineering and Informatics, Technical University of \\ Košice, Letná 9, 04200 Košice, tel. 055/602 4214, E-mail: jakub.cerkala@tuke.sk, anna.jadlovska@tuke.sk
}

\begin{abstract}
This article focuses on mathematical modelling of mobile robot with differentially driven two-wheel chassis. The derivation of kinematic model is based on holonomic constraints for it's wheels movement, that are expressed through geometric transformations between robot's local coordinate system and the global coordinate system, in which the robot is moving. Within the dynamics derived using the Newton's laws, the friction between the contact surfaces of wheels and plane is also included as a generalized model. Mathematical model is implemented in the Simulink environment with internal control loop that suppress the impacts of dynamics. Conducted simulation experiments in open and closed-loop demonstrate, how the inclusion of the friction can have serious impact on the mobile robot final position in meaning of the forces, which are acting on it's movement.
\end{abstract}

Keywords: mobile robot, control, trajectory tracking, kinematics, mathematical modeling, simulation, Matlab/Simulink

\section{INTRODUCTION}

In recent years, the mobile robotics has seen a significant development from a scientific perspective, but also in terms of expansion to business or to general public [1]. The logistic, service or social robots of nowadays that are moving in plane usually use the differential driven two-wheels chassis concept because it offers a high mobility with good stability and this article focuses mainly on their mathematical modelling. Moreover, the two-wheel differential chassis concept is the base for more complex multi-wheeled or tracked chassis robot designs. Basic mathematical models of mentioned types of robots can be divided into the independent kinematic model and the dynamic model, which usually have the internal control loop designed to suppress the impacts of dynamics [2].

In order to obtain the kinematics model, the holonomic constraints for the mobile robot overall movement has to be taken into account. These constraints can be derived using the chassis geometric properties and transformations between the coordinate systems [3], this article presents the general approach to obtain holonomic constraints based on the wheel position's on the chassis. Dynamical properties of the mobile robot can be obtained using Newton-Euler method or using the Lagrange approach [3], however both approaches doesn't include the friction, which affects the moving robot.

The friction is complex non-linear physical phenomenon [4], [5]. In the most cases, is usually considered as a disturbance and the goal of the system control is to eliminate it's effects. In mobile robotics, the friction is often neglected [3], owing to the size of robot, overdimensioned actuators and internal control loops. In some cases, the friction is considered only as a simple model [2], but in terms of forces that are really affecting the robot's movement it plays a significant role. In this article, a general model of friction is used as an extension of the mobile robot dynamics in conjunction with [6].

Mathematical model of the mobile robot is implemented in simulation environment Simulink [7], where the non- linear model of general friction can be included into the robot's simulation model which can be used in experiments. Although friction affects mainly the dynamic properties of the robot and the velocities of the wheels, to get a better idea of it's impacts, it is appropriate to preview them as differences of robot's movement in plane using the kinematic model of the mobile robot.

\section{MATHEMATICAL MODEL OF NONHOLO- NOMIC MOBILE ROBOT WITH DIFFEREN- TIAL TWO-WHEEL CHASSIS}

Mathematical model of the mobile robot, which is used as an approximation of the real mobile robot consists of kinematic model and dynamic model [2], [6]. To suppress the robot's dynamics, an internal loop is also included - the block scheme of the mobile robot is depicted on Fig. 1this robot can be used further in control structures.

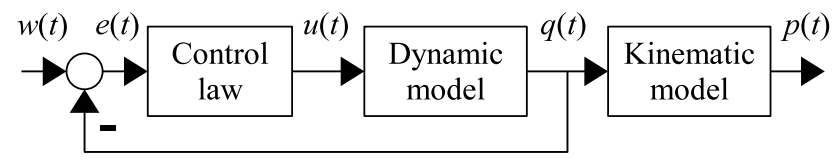

Fig. 1 Mobile robot with internal control loop

The mobile robot's reference input $w(t)$ are desired velocities, the output of internal control loop $q(t)$ are robot's current velocities, which are then by kinematic model transformed to resulting robot posture $p(t)$. The control inputs are differences between the desired and current velocities $e(t)=w(t)-q(t)$ while the control output $u(t)$ affects the dynamics of the robot as forces or torques. Posture of the robot in respect to origin of the global coordinate system (GCS) is expressed by position coordinates $x, y$ of it's local coordinate system (LCS) origin with rotation defined by an angle $\varphi$. At the same time, the position vector $p$ can include the angular rotation position of robot wheels as $\theta_{R}, \theta_{L}$, but they have rather informational value. 
Position vector of the mobile robot is defined as

$$
p_{\text {robot }}=\left[\begin{array}{lllll}
x, & y, & \varphi, & \theta_{R}, & \theta_{L}
\end{array}\right]^{\mathrm{T}}
$$

The kinematic model of two-wheeled mobile robot with differentially controlled motors, which chassis concept scheme is depicted on Fig. 2, is defined for LCS origin $O_{L C S}$, that usually lies in the middle of rotation axis between wheels. The distance from $O_{L C S}$ to wheel mount is $\pm b$ and both wheels have the same radius of $r$. In this article, the point $O_{L C S}$ is also the centre of gravity and the reference point for mobile robot control.

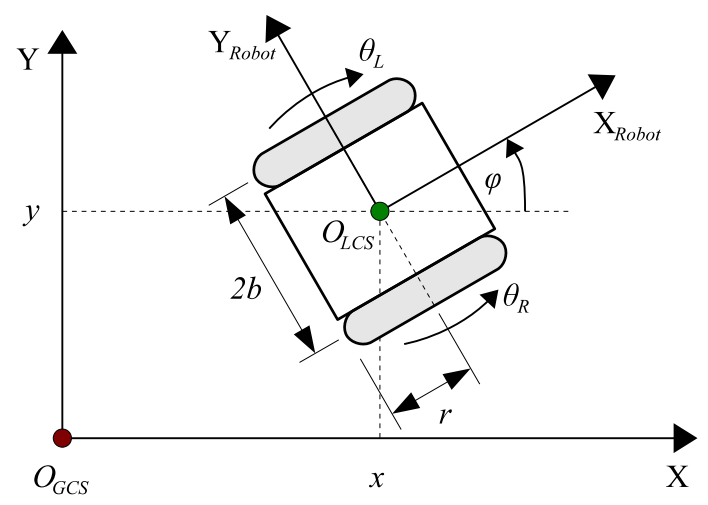

Fig. 2 Mobile robot in plane

Kinematic model can be defined for robot's overall linear velocity $v(t)$ and it's angular velocity $\omega(t)$, but for the most control structures [1], [2] it is necessary to define it for wheel angular velocities $\omega_{R}(t), \omega_{L}(t)$. For any oriented point (posture) defined by coordinates $\xi^{L}=\left[x_{i}^{L}, y_{i}^{L}, \varphi_{i}^{L}\right]^{\mathrm{T}}$ in robot's LCS, it is possible to obtain it's posture in GCS with respect to the initial position of $O_{L C S}$ as $\xi^{G}=\left[x_{i}, y_{i}, \varphi_{i}\right]^{\mathrm{T}}$ using the rotation transformation

$\xi^{G}=R(\varphi) \xi^{L}$

while the rotation matrix $R(\varphi)$ is defined for positive, counter-clockwise rotation

$R(\varphi)=\left[\begin{array}{ccc}\cos \varphi & -\sin \varphi & 0 \\ \sin \varphi & \cos \varphi & 0 \\ 0 & 0 & 1\end{array}\right]$

Also, the same transformation of coordinates applies on point velocities as

$\dot{\xi}^{G}=R(\varphi) \dot{\xi}^{L}$

While deriving the kinematic model, the holonomic constraints dependent on chassis dimensions and wheel placement has to be taken into account.

\subsection{Holonomic constraints for mobile robot movement}

For the mobile robot kinematics, it is assumed that robot wheels are rolling without the lateral and longitudinal slip [1], [3]. Velocity of the robot in terms of $y$-coordinate in robot's LCS is zero and using rotation matrix $R(\varphi)$ it is possible to define holonomic constraint for the robot position in GCS as

$\dot{y}^{L}=0 \quad \longrightarrow \quad[0, \quad 1, \quad 0] \cdot R(\varphi)^{-1} \dot{\xi}^{G}$,

which gives

$-\sin (\varphi) \dot{x}+\cos (\varphi) \dot{y}=0$

The same (6) constraint for lateral movement applies on both wheels and it can be obtained from general definition of classical wheel placement in chassis. The position of wheel in robot's LCS can be defined using polar coordinates as demonstrated on Fig. 3 , where $l$ is euclidean distance between $O_{L C S}$ and wheel mount, $\alpha$ denotes the angle of the mount against LCS X axis and the $\beta$ defines the shift in wheel mount.

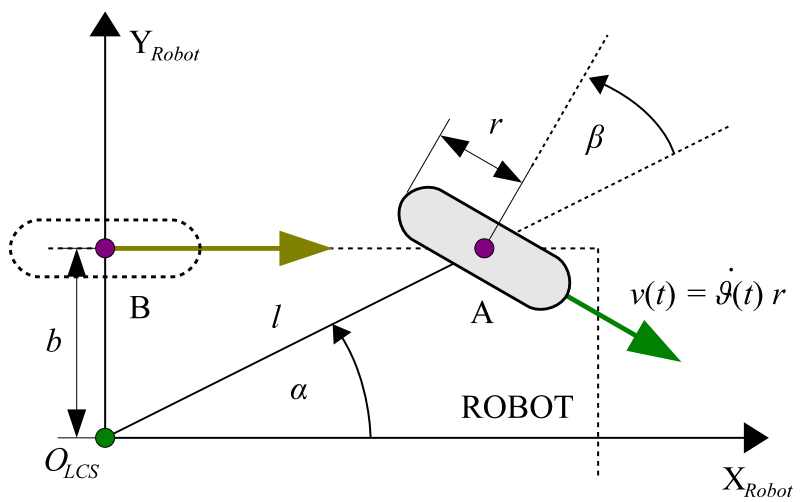

Fig. 3 General position definition of robot wheel (A) and left wheel position definition (B)

Based on the geometric properties of the wheel mounted in point $\mathrm{A}$, the constraint for zero movement in LCS Y axis can be defined in GCS as

$[\cos (\alpha+\beta), \quad \sin (\alpha+\beta), \quad l \sin (\beta)]_{A} R(\varphi)^{-1} \dot{\xi} G=0$

where for values $\alpha=\pi, \beta=0$ and $l=b$ that applies for left wheel mounted in point $B$, gives

$\dot{y}_{B}^{L}=0 \quad \longrightarrow \quad[0, \quad 1, \quad 0]_{B} \cdot R(\varphi)^{-1} \dot{\xi}^{G}$

which leads to the same result as in (6) as well in the case of right wheel.

Since both wheel are rolling without the longitudinal slip (pure rolling), the linear velocities in can be expressed as

$v_{R}(t)=r \dot{\theta}_{R}, \quad v_{L}(t)=r \dot{\theta}_{L}$.

The constraint for wheel mounted in point $\mathrm{A}$ in terms of LCS $\mathrm{X}$ axis can be expressed in GCS as

$[\sin (\alpha+\beta),-\cos (\alpha+\beta),-l \cos (\beta)]_{A} R(\varphi)^{-1} \dot{\xi} G=r \dot{\vartheta}$

where $r$ is wheel radius and $\dot{\vartheta}$ is wheel's angular rolling velocity. As previous, for the left wheel with the same values $l, \alpha, \beta$ as in 8 and $\dot{\vartheta}=\theta_{L}$ is

$\left[\begin{array}{lll}1, & 0, & -b\end{array}\right]_{B}\left[\begin{array}{ccc}\cos \varphi & \sin \varphi & 0 \\ -\sin \varphi & \cos \varphi & 0 \\ 0 & 0 & 1\end{array}\right]\left[\begin{array}{l}\dot{x} \\ \dot{y} \\ \dot{\varphi}\end{array}\right]_{G}=r \dot{\vartheta}$ 
the another holonomic constraint defined as

$\cos (\varphi) \dot{x}+\sin (\varphi) \dot{y}-b \dot{\varphi}-r \dot{\theta}_{L}=0$.

The same approach applies also for right wheel, that differs in $l=-b$ and overall holonomic constraints for mobile robot movement (6), (12) can be expressed as matrix defined for position vector (1) as

$$
\left[\begin{array}{ccccc}
-\sin (\varphi) & \cos (\varphi) & 0 & 0 & 0 \\
\cos (\varphi) & \sin (\varphi) & b & -r & 0 \\
\cos (\varphi) & \sin (\varphi) & -b & 0 & -r
\end{array}\right]\left[\begin{array}{c}
\dot{x} \\
\dot{y} \\
\dot{\varphi} \\
\dot{\theta}_{R} \\
\dot{\theta}_{L}
\end{array}\right]=0
$$

According to these holonomic constraints (13), it is possible to derive the kinematic model for the mobile robot chassis in following part of the article.

\subsection{Kinematic model of mobile robot}

The general kinematic model of mobile robot defined for overall linear velocity $v=\dot{x}^{L}$ and overall angular velocity $\omega=\dot{\varphi}$ can be derived using $R(\varphi)$ in GCS as

$$
\left[\begin{array}{c}
\dot{x} \\
\dot{y} \\
\dot{\varphi}
\end{array}\right]=\left[\begin{array}{cc}
\cos (\varphi) & 0 \\
\sin (\varphi) & 0 \\
0 & 1
\end{array}\right]\left[\begin{array}{c}
v \\
\omega
\end{array}\right]
$$

As mentioned previously, for the control purposes it is often required to define kinematic model for wheel velocities. Overall linear velocity of the robot is mean value of both wheel linear velocities expressed as

$v=\frac{v_{R}+v_{L}}{2}$

Overall angular velocity in term of instantaneous centre of curvature (ICC) can be expressed as

$\omega=\frac{v_{R}}{R_{0}+b}, \quad$ respectively $\quad \omega=\frac{v_{L}}{R_{0}-b}$,

where by substitution of actual turning radius $R_{0}$, the equation for overall angular velocity expressed by wheel linear velocities $v_{R}, v_{L}$ can be obtained as

$\omega=\frac{v_{R}-v_{L}}{2 b}$

On the basis of relations (9), it is possible to modify (15), 17) for angular velocities as

$v=r \frac{\omega_{R}+\omega_{L}}{2}, \quad \omega=r \frac{\omega_{R}-\omega_{L}}{2 b}$

The kinematic model defined for position vector (1) and wheel angular velocities $\omega_{R}, \omega_{L}$ is obtained by substitution of (18) into general kinematic model (14) while it satisfies the holonomic constraints (13) and it can be written in compact matrix form

$$
\left[\begin{array}{c}
\dot{x} \\
\dot{y} \\
\dot{\varphi} \\
\dot{\theta}_{R} \\
\dot{\theta}_{L}
\end{array}\right]=\left[\begin{array}{cc}
\frac{r \cdot \cos (\varphi)}{2} & \frac{r \cdot \cos (\varphi)}{2} \\
\frac{r \cdot \sin (\varphi)}{2} & \frac{r \cdot \sin (\varphi)}{2} \\
\frac{r}{2 b} & -\frac{r}{2 b} \\
1 & 0 \\
0 & 1
\end{array}\right]\left[\begin{array}{c}
\omega_{R} \\
\omega_{L}
\end{array}\right]
$$

The kinematic model (19) as mobile robot representation is standalone applicable for some control structures, but the inclusion of dynamics will increase it's accuracy.

\subsection{Dynamic model of mobile mobile robot}

Dynamic model of mobile robot presented in this article includes the effects of robot's overall mass $m$ and overall moment of inertia $J$. It can be obtained using the second Newton's law [6] for forces

$F_{i}=m a_{i}$

In application of 20 to wheel motor's traction forces $F_{R}$, $F_{L}$, it is possible to express the overall angular acceleration as

$\dot{v}=\frac{F_{R}}{m}+\frac{F_{L}}{m}$

Traction forces $F_{R}, F_{L}$ can be defined also as wheel torques, while the overall torque of the robot is defined as a sum of wheel partial torques $\tau_{R}=F_{R} b, \tau_{L}=-F_{L} b$ as

$\tau_{\text {Robot }}=\tau_{R}+\tau_{L}$

where opposite direction of $F_{L}$ is required to satisfy the equation (22). Again, using the second Newton's law, it is possible to express $\tau_{\text {Robot }}$ as function of $\dot{\omega}$ as

$\tau_{\text {Robot }}=J \dot{\omega}$,

where the $J$ is robot's overall moment of inertia. Angular acceleration $\dot{\omega}$ can be also expressed as a function of traction forces

$\dot{\omega}=\frac{F_{R} b}{J}-\frac{F_{L} b}{J}$

Based on the relations (21), 24, it is possible to obtain the mobile robot's dynamic model defined for wheel motor's traction forces $F_{R}, F_{L}$, that can be implemented in simulation model of mobile robot (Fig. 1).

\subsection{Internal control loop}

Internal control loop serves for the suppression of the mobile robot dynamics impacts. The control target is to minimize the error between the desired and actual velocities of the robot using traction forces as control outputs. In the case of considering only the mass and inertia, it is possible to apply the $\mathrm{P}$ controllers, however, in most cases, where there are disturbances, it is necessary to use PI controller to avoid permanent control deviation. Also, the DC motors, gearboxes and encoders with own dynamics are included in control loop, but in this article they are assumed as transfer function of 1 for the simplification. However, the control output have to be limited for the maximal traction force, that the wheel motor can produce.

Mathematical model of mobile robot consisting of kinematic model (19) and dynamic model (21), (24) with control loop can be implemented as simulation model in Simulink environment. 


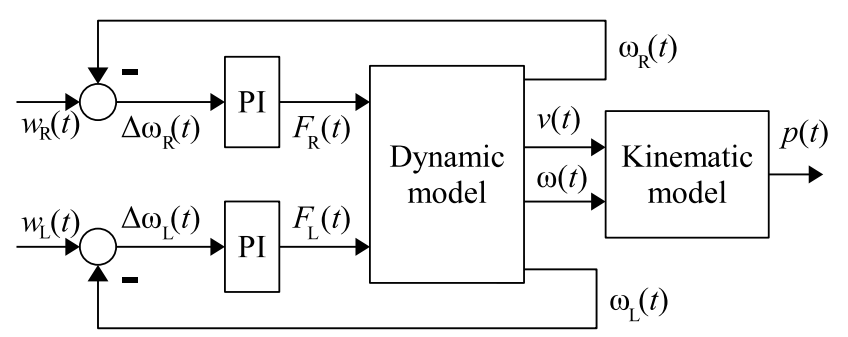

Fig. 4 Mobile robot's internal control loop in detail

The behaviour of this model is much closer to real model than the kinematic model standalone, but it is still inaccurate in terms of traction forces $F_{R}, F_{L}$ actuation. In case of motion with constant velocity, when there is no acceleration, the traction forces are no longer acting. In real mobile robot, to maintain it's the constant velocity requires the constant actuation, which compensates the effect of gravitational pull and friction between contact surfaces. Therefore, it is appropriate to include the friction effects into simulation model of mobile robot to achieve better accuracy.

\subsection{Friction as dynamics extension}

In most cases, the friction is expressed as dependency of force acting against moving object to it's velocity and the most used mathematical models of friction are depicted on Fig. 5. Basic friction models include Coulomb (kinetic) friction, viscous friction and static friction (stiction) with Stribeck effect [4], [5].

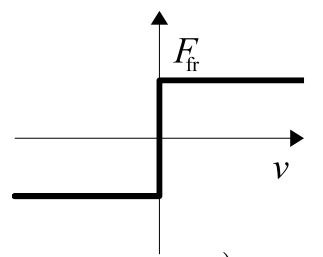

a)

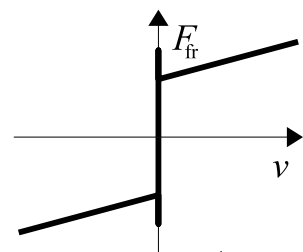

c)

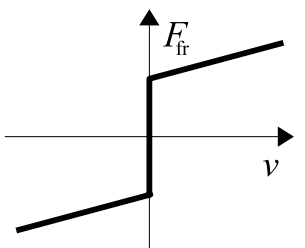

b)

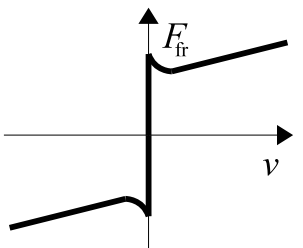

d)
Fig. 5 Common friction models: a) Coulomb,

b) Coulomb + viscous,

c) Coulomb + viscous + stiction,

d) Coulomb + viscous + Stribeck effect

Coulomb friction model defines the constant friction force against movement as

$F_{k}=\mu_{k} F_{N} \cdot \operatorname{sgn}(v), \quad$ where $F_{N}=m g$,

and $\mu_{k}$ is Coulomb friction coefficient, $F_{N}$ is normal force acting on robot and $\operatorname{sgn}(v)$ defines the direction of $v$. If the robot is moving $(v \neq 0)$, it is possible to apply the viscous friction as

$F_{v}=\mu_{v} v$

where $\mu_{v}$ is viscous friction coefficient and it rises with rising velocity. If the robot doesn't move $(v=0)$, it is still affected by the static friction, often known as stiction which expresses the force $F_{s}$, that excitation force $F_{e}$ need to overcome to set robot moving and it can be defined as

$F_{s}=\left\{\begin{array}{ccc}F_{e} & \text { for } & \left|F_{e}\right|<\mu_{s} F_{N} \operatorname{sgn}\left(F_{e}\right), \\ \mu_{s} F_{N} \cdot \operatorname{sgn}\left(F_{e}\right) & \text { for } & \left|F_{e}\right| \geq \mu_{s} F_{N} \operatorname{sgn}\left(F_{e}\right),\end{array}\right.$

where $\mu_{s}$ is static friction coefficient. It always applies that $\mu_{s} \geq \mu_{k}$ and in non-equal case $\left(\mu_{s} \neq \mu_{k}\right)$ with nonzero low velocity, the Stribeck effect applies - it defines the curve shape of the exponential friction decrease with increasing velocity by coefficient $c_{s}$. The kinetic friction, acting against moving robot can be considered as

$F_{f r}(v)=F_{k}+\left(F_{s}-F_{k}\right) e^{-c_{s} \cdot|v|}+F_{v}$

The general model of friction based on Coulomb (25), viscous (26) and static friction 27) with Stribeck curve 28) can be expressed as

$F_{f r}\left(v, F_{e}\right)=\left\{\begin{array}{ccc}F_{e} & \text { for } v=0 \wedge F_{e}<F_{s}, \\ F_{s} \cdot \operatorname{sgn}\left(F_{e}\right) & \text { for } v=0 \wedge F_{e} \geq F_{s}, \\ F_{f r}(v) & \text { for } v \neq 0\end{array}\right.$

\subsection{Mathematical model implementation in Simulink}

Since it is that the friction force $F_{f r}$ acts against robot movement or against the excitation force $F_{e}$ while not moving, it must be ensured that the friction force wouldn't cause the movement of the robot already in the implementation of model (Fig. 4) into the simulation environment. It is possible using the State and Reset integrator ports of (21), 24) as shown on Fig. 6

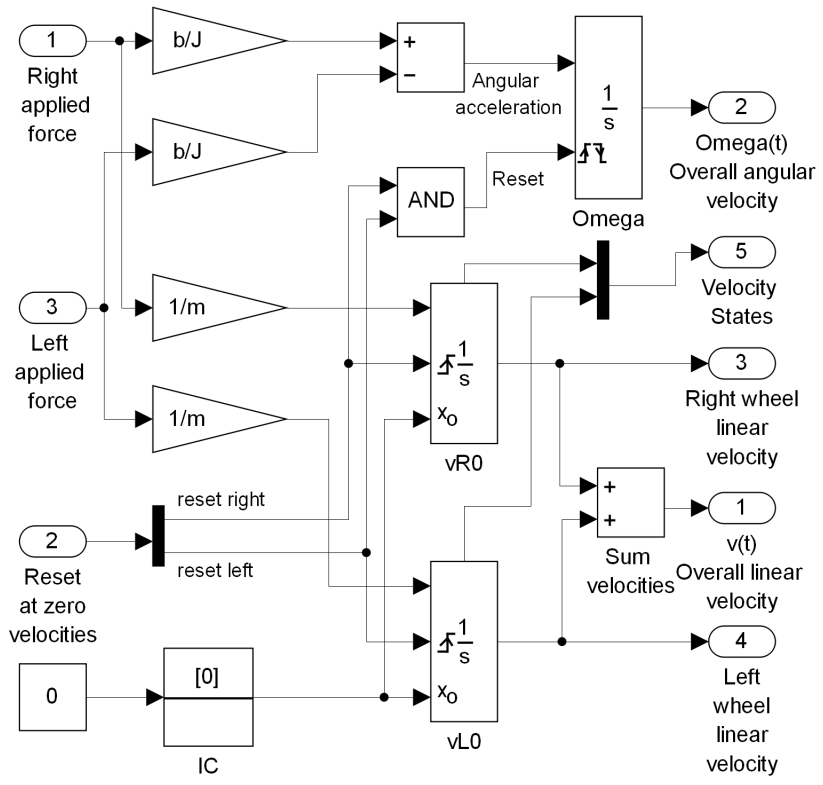

Fig. 6 Implementation of dynamic model as subsystem in Simulink 
Using the Hit crossing block on the output of integrators state ports, the future transition of wheel linear velocity from and to zero is captured, which will trigger the friction force - static or kinetic, wherein a reset of the wheel linear velocity occurs only when there is only a friction force acting and slowing the non-actuated motion of the wheel. At the same time, it is necessary to reset the overall angular velocity integrator in case, when both wheels are not moving.

\section{SIMULATION RESULTS}

To verify how friction affects the movement of mobile robot, the open-loop without the internal control loop and closed loop-control simulations were carried out. Dynamic parameters of mobile robot used in both simulation experiments are: $m=0.5 \mathrm{~kg}, J=5 \cdot 10^{-4} \mathrm{~kg} \cdot \mathrm{m}^{2}$ and friction coefficients are $\mu_{K}=0.05, \mu_{S}=0.05, \mu_{V}=0.1$ and $c_{s}=20$.

\subsection{Open-loop verification}

The first experiment depicted on Fig. 7 demonstrates, how the mobile robot is stopped due to friction in comparison to the robot with same dynamics, but no friction - it remains in constant circular movement. The excitation force $F_{e}$, that rises from zero in time $t=0 \mathrm{~s}$ with trapezoidal shape with $0.5 \mathrm{~N}$ maximum is fed to the inputs of dynamic model as traction forces $F_{R}, F_{L}$. The left wheel receives the $10 \%$ lower input traction force to achieve final circular trajectory. In time $t=1.5 \mathrm{~s}$ the force $F_{e}$ fall back to zero.

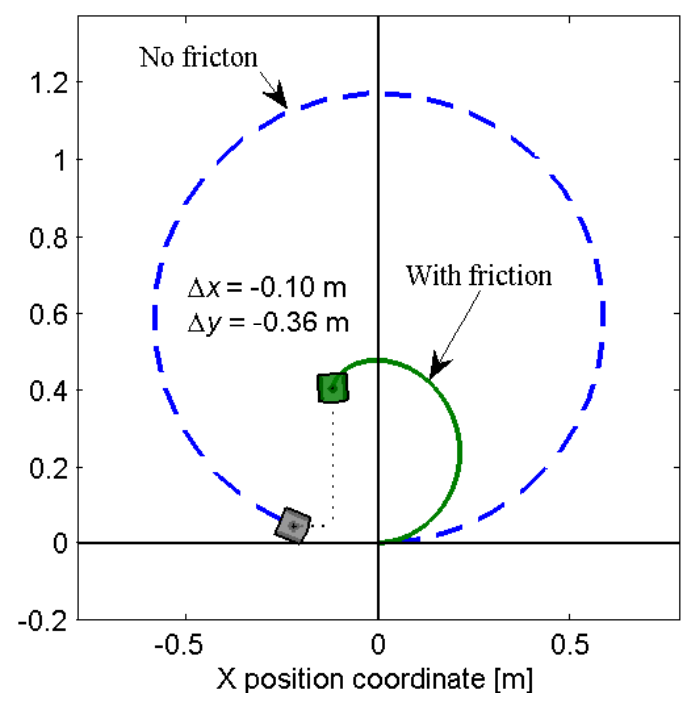

Fig. 7 Open-loop experiment - difference between dynamics with and without friction in plane motion

The action of the friction components $F_{f r R}$ to right wheel linear velocity are shown on Fig. 8. Without any input, the robot slows down due to friction.

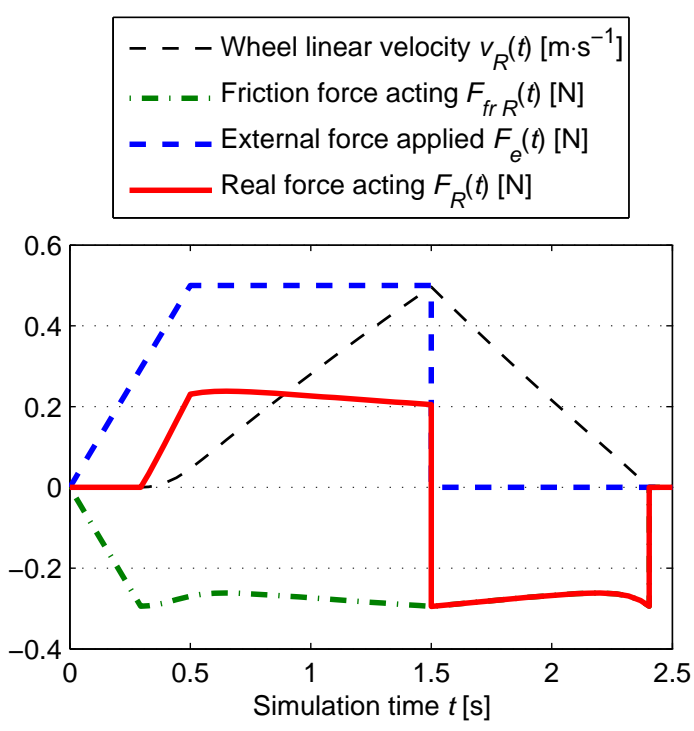

Fig. 8 Open-loop experiment - forces affecting right wheel's velocity

\subsection{Closed-loop verification}

The second experiment depicted on Fig. 9 compares the relative position of kinematic (ideal) model towards the models with dynamics and internal control loop in desired movement on unit circle. Internal loop PI controllers ensure zero control deviation in terms of the desired overall linear velocity $v_{w}=0.5 \mathrm{~m} / \mathrm{s}$ and overall angular velocity $\omega_{w}=0.5 \mathrm{rad} / \mathrm{s}$, but at a cost in terms of position displacement. In the case of dynamics with friction, the control input in steady state is constant and non-zero, the plot of forces is acting on right wheel shown in Fig. 10.

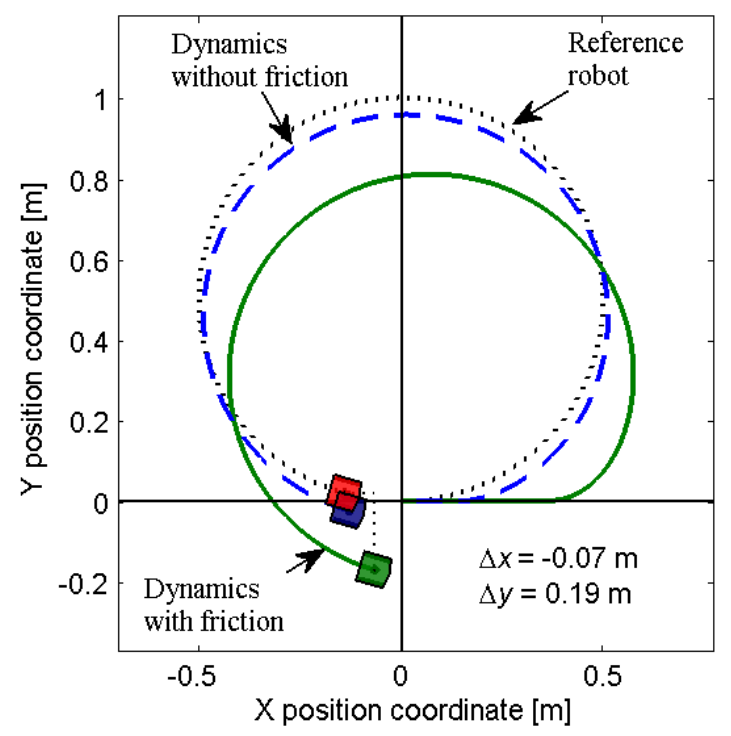

Fig. 9 Closed-loop experiment - difference between dynamics with and without friction in plane motion 


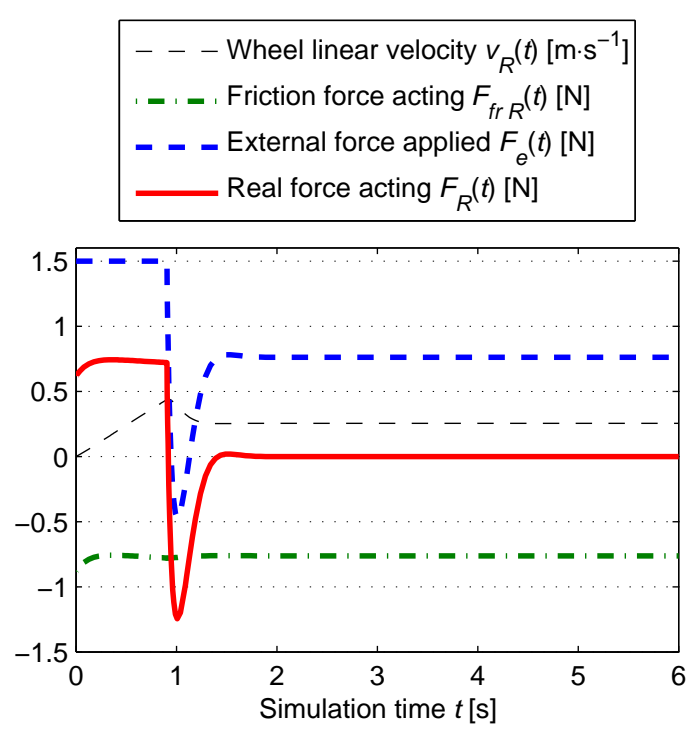

Fig. 10 Closed-loop experiment - forces affecting right wheel's velocity

\section{CONCLUSIONS}

The main focus of this article is to present the approach to obtain the mathematical model of differentially driven two-wheel mobile robot, that consist of kinematic model and dynamic model with internal control loop using Newton's laws approach. This approach is extended with chassis constraints analysis, that can be applied also in the kinematics model derivation of different wheel configurations. Moreover, the general model of friction is also included into dynamics of mobile robot's simulation model in Simulink environment to increase model accuracy. The presented experiments demonstrate, how the friction can affect dynamics of the robot in terms of it's position. The obtained simulation model can be further used as a source of more accurate training data in application of neural models in control structures for mobile robot control tasks to achieve a more realistic behaviour [2].

\section{ACKNOWLEDGEMENT}

This work has been supported by the Research and Development Operational Program for project: University Science Park Technicom for innovative applications with knowledge technology support, ITMS code 26220220182 , co-financed by the ERDF $(80 \%)$ and by grant KEGA 001TUKE-4/2015 (20\%).

\section{REFERENCES}

[1] SIEGWART, R. - NOURBAKHSH, I. R. - SCARAMUZZA, D.: Introduction to autonomous mobile robots. MIT press, 2. edition, 2011, ISBN 978-0-26201535-6

[2] ŠUSTER, J. - JADLOVSKÁ: Tracking trajectory of the mobile robot Khepera II using approaches of ar- tificial intelligence In: Acta Electrotechnica et Informatica 11, No. 1, 2011, pp. 38-43, ISSN 1335-8243

[3] DHAOUADI, R. - HATAB AA.: Dynamic Modelling of Differential-Drive Mobile Robots using Lagrange and Newton-Euler Methodologies: A Unified Framework In: Adv Robot Autom 2, No. 107, 2013, ISSN: 2168-9695, doi: 10.4172/2168-9695.1000107

[4] IURIAN, C. et al.: Identification of a system with dry friction, External research report, 2005, http: //hdl.handle.net/2117/511

[5] VIRGALA, I. - KELEMEN, M.: Experimental friction identification of a DC motor. In: International Journal of Mechanics and Applications 3, No.1, 2013, 26-30, ISSN: 2165-9303, doi:10.5923/j.mechanics.20130301.04

[6] ČERKALA, J. - JADLOVSKÁ: Dynamics with Friction in Mobile Robot Simulink Model In: Technical Computing Bratislava 2014 Proceedings. Saarbrücken : Lap Lambert Academic Publishing, 2015, pp. 65-81, ISBN 978-3-659-40792-5

[7] ŠEMBERA, J. - ŠOLC, F.: Modelling and Control of Differential Drive Robot In: ATP Journal, 2007, pp. 203-207, ISBN 978-80-8073-874-7

Received July 22, 2015, accepted November 11, 2015

\section{BIOGRAPHIES}

Jakub Čerkala was born February 4th, 1988. In 2012 he graduated (MSc.) with distinction at the Department of Cybernetics and Artificial Intelligence of the Faculty of Electrical Engineering and Informatics at Technical University in Košice. Since September 2012 he has been internal PhD. student at the Department of Cybernetics and Artificial Intelligence. The topic of his dissertation thesis is focused on mobile robotics and Artificial intelligence methods, a rather different field than his previous study oriented on experimental identification and hydraulics systems. He is also included in various research projects.

Anna Jadlovská was born October 29th, 1960. She received her MSc. degree in the field of Technical Cybernetics at the Faculty of Electrical Engineering of the Technical University in Košice in 1984. She defended her PhD thesis in the domain of Automatization and Control in 2001 at the same University; her thesis title was Modelling and Control of Non-linear Processes Using Neural Networks. Since 1993 she worked in the Department of Cybernetics and Artificial Intelligence Faculty of Electrical Engineering and Informatics Technical University in Košice as an Associate Assistant and since 2004 she has been working as an Associate Professor. Her main research activities include the problems of adaptive and optimal control in particular predictive control with constraints for non-linear processes using neural networks and methods of artificial intelligence (Intelligent Control Design). She is the author of scientific articles and contributions to various journals and international conference proceedings, as well as being the co-author of some monographs. 